\title{
Use of Triexta fabric in fashion garment as stain repellent fabric
}

\begin{abstract}
Stain removing or dirt cleaning is a tedious job during activity of day to day garment washing. This dirt is held fast due to mixing of oil secreted from body. During washing it is very difficult to clean these parts and in due course these part turns yellow due to left over residue after washing. To solve this problem garment designers and textile technologists are working continuously till now. Users will get relieve during cleaning if the garment itself is clean friendly. A common popular technique has been adopted, by treating the fabric before pattern to be cut, with chemicals during functional finishing process. But this step creates a lot of difficulties during garment making process including high rise in cost due to an extra application. Garments are made only to focus about the aesthetic appeal of the dress and not to think there after use care. This may possible only for couture or avant grade item and not for in day to day life. Triexta is such a fibre having inherent property to repel soil and dirt adhere to it naturally during washing. Considering this property and its bio-friendly manufacturing process, using of Triexta fabric in the garment making as one of the raw materials in some strategic parts is done in our studies.
\end{abstract}

Keywords: Triexta fabric, fashion garment, textile, purified terepthalic acid, polyester
Volume 5 Issue 5 - 2019

\section{Mainak Mitra}

Department of Jute and Fibre Technology, Calcutta University, India

Correspondence: Mainak Mitra, M tech, Department of Jute and Fibre Technology, Calcutta University, India, Tel +919674609788, Emailmmmtech2013@gmail.com

Received:September 15, 2019 | Published: September 30, 2019
Abbreviations: PTT, poly-trimethylene terephthalate; FTC, federal trade commission; PTA; purified terepthalic acid; PDO, 1, 3 propane-diol; PET; polyester; VOC, volatile organic compounds

\section{Introduction}

Triexta is invented by Dupont, who was the inventor of Nylon also. Presently the sole producer of Triexta is Dupont and sells in the brand name of Sarona. Mohawk Industries is also marketed Sarona in the brand name of Smart-Strand. Mohawk is the largest manufacturer of this fibre mainly supplies to carpet industry as raw material. The technical name of Triexta is Poly-trimethylene terepthalate, abbreviated as PTT. ${ }^{1}$

The mother chemical of both Triexta and Polyester are same. So initially Triexta was named as Polyester. Dupont along with Mohawk Industries showed the significant differences in the final product and then they termed triexta as a new generic name PTT. The U.S. Federal Trade Commission (FTC) in the year 2009 officially gave recognition Triexta as a subclass of polyester and generically termed PTT.

Polyester is more lustrous and gives a shiner look than other fibres, whereas Nylon has comparatively more matte finish. From the appearance point of view Triexta has more similarity to nylon than polyester. $^{2}$

It is one of the challenges for the cleaners to identify the polyester from Triexta during sorting. Generally the initial fibre identification is done by Burning test. But during burning both the fibres give an orange sputtering flame with residue a black sooty smoke. The inflame property of nylon and olefin group fibres is orange tinge with blue base and producing white puff of smoke when away from flame. The distinguishing test which separates polyester from Triexta is fluoro-chemical protection test. In this test a drop of baby oil is gently placed on the yarn with the rim of the bottle just touching the yarn.
Polyester behaves a proclivity to bead the oil drop whereas Triexta ${ }^{3}$ readily soaks it.

The thermoplastic fibre PTT or polytrimethylene terepthalate can be spun into yarn and popularly are used in carpet industry as raw material. The fibre synthesis is done by poly-condensation of PTA (purified terepthalic acid) and PDO (1, 3 propane-diol), chemically/ biochemically manufactured by Shell Group and Dupont. From the physical property point of view the fabrics and yarns made from this fibre are strong, having high abrasion resistance, elastic (return back into shape) and wrinkle free nature.

The difference in superiority of PTT fibre to PET polyester is clearly observed in the following properties:

1. Durability: Like nylon PTT is more durable than PET

2. Hand: PTT is softer and smoother to touch than PET

3. Effect of stain \& care: Triexta fibre is hydrophobic \& good resistant to stain. So without using any spot cleaner fabric can be washed easily only by water. To some common household foods like red wine, coloured beverages, mustard, PTT is resistant to stain permanently. ${ }^{4}$ No additional chemical treatments are required for stain removal as it is self resistant to stain. Due to its poor moisture absorbent property triexta fabric apparel was popular in clothing industry earlier than carpet industry with the trade tag-line 'dry-fit'. Like PET and olefin PTT is oleophilic, so soils (petroleum, animal, vegetable) and oil spots can easily be removed by applying dry solvent spotters and cleaning additives, like propylene glycol.

4. Effect of microorganism: PTT is allergy friendly, mold resistant like other synthetic fibres, so it is safe during handling by kids. 
5. Sustainability \& eco friendliness: To produce an equal amount of nylon- 6 , PTT requires $30 \%$ less energy and $63 \%$ reduction in green house gas emission. PTT establishes its environment friendly status by consuming $30 \%$ less energy as compared to nylon- 6 and $40 \%$ less energy as that of nylon-6.6.

6. Bio production: An attempt of using biotechnology by Dupont the cost of production becomes lower down. It is done through the fermentation of corn glucose by replacing 37\% use of petroleum raw material to produce Dupont Sarona. Corn glucose is a renewable source and much more sustainable than petroleum. Also inclusion of corn glucose as raw material by replacing petroleum is a result of lesser chemicals input in the fibre manufacturing, which in turn relates to evolution of fewer chemicals as volatile organic compounds (VOC) known as offgassing.

7. Both PTT fibres \& yarn have good stretch recovery, better softness, good bulk property and easy dye-ability.

8. Effect of bleaching: PTT has greater resistant to strong oxidizing bleaches, viz. concentrated (up to 6\%) sodium hypochlorite

9. Colour fastness: PTT has better fastness property over PET \& nylon-6 \& 66 .

10. Hand feel: Triexta is softer than both nylon and polyester due absence of chemical treating for stain protection during fibre manufacturing.

11. Loop Berber style: During putting of pressure on underfoot fabric we feel roughness if made from nylon or olefin but this tendency is absent in case of Triexta, making it feather to touch and comfortable underfoot.

12. Effect of dyeing: Amongst the various ways of dyeing the popular technique for Triexta is using disperse dye through continuous process.

13. Effect of sunlight: Triexta is fade resistant, so direct exposure to sunlight or UV light results no discoloration, being suitable for furnishing fabric.

14. Costing: Triexta is very much pricing competitive due to its low cost of production. Actually the price of Triexta is in between nylon and polyester.

15. Engineered property: Mohawk emphatically says that 'Smartstrand' is chemically engineered as stain resistant and not by coating. In their website they blazon that 'Smart-strand' attains the height of anti-spattering by removing tough stain, like red wine, cherry, mustard etc. only with water and mild detergent. They are officially announcing regularly that the 'Smart-strand' is resistant to discoloration during bleaching for removal of children soiling, carpeted kitchen and frequent spills.

\section{Uses}

Abundant examples are found about the application of Triexta fabrics in apparel, home furnishing and automotive industry as premium quality carpets, sheets pillow cases, upholstery, floor mats, automotive interiors, premium swimwear and active wear.

\section{Our study}

Keeping in the mind of its eco-friendliness and inherent stain resistant property we select Triexta as raw material for our term garment. The objectives behind the project were searching for sustainable \& renewable source and less caring hazard during daily use and storage. The garment is engineered for cook, working personnel in dusty atmosphere, children etc.

We use cotton muslin fabric, Triexta fabric and polyester sewing thread as raw materials for making of garment. We have made full sleeve shirt with cotton fabric as main garment material and Triexta fabric at collar, calf, under arm seam and placket.

$120 \mathrm{gsm}$ cotton fabric and $250 \mathrm{gsm}$ Triexta fabric were used. Higher gsm selected for Triexta is to get better drape at those strategic parts of the garment.

Fabric was not treated with any functional finishing agent before and after the garment making, i.e. with virgin fabric only.

Comparing the cost of $100 \%$ cotton shirt and this blended functional fabric, it is easily understood that our fabric is much cheaper than the virgin cotton shirt. We used $15 \%$ fabric as Triexta, so cost is reduced proportionately, as price of Triexta is much lesser than pure cotton fabric.

\section{Testing of stain removal}

To get an extreme stained condition we put the fabric in domestic birds enclose and on kitchen floor as foot mat for a week, encouraging them to spill excretion and condiments on the fabric. After a week we took the spoiled fabric under hot water splash followed by putting in normal detergent water solution for half an hour And rinse thoroughly. The fabric got previous look leaving no trace of any stain.

\section{Disadvantages of functional finished stain removed garments}

a. Extra cost of manufacturing due to an extra finishing process. Sometimes users are not ready to pay that.

b. Some changes are to incorporate during manufacturing regarding interfering, lining, and new tape compatible to the fabric in terms of shrinkage.

c. To avoid seam puckering machine tension, stitching techniques to be adjusted.

d. Soil and stain resistant finishes sometimes alter the fabric surface energy and behavior during interplaying with dirt showing a newer home laundering process. Study reveals that textile softeners viz. waxes, silicones, anti-crease chemicals fatty acid derivatives have shown unsatisfactory stain removing performance as well as poor whiteness index. Theory supports when amino-silicone finished fabrics produce poor stain removal index for oil spots with low whiteness property. This can be explained by their newly developed hydrophobic property and behavior with stains. It can also be justified that fabric finished with nonionic fatty amide produces better stain removal profile due to its newly developed hydrophilic surface but less finish wash fastness. The more the whiteness index betters the brightener fastness. ${ }^{5}$ 
All the above makes the manufacturing process disturbed and sometimes faulty.

\section{Hazards facing during removal of stain in washing}

i. Before using the stain removing agent it is to be tested at the hidden part or on seam allowance of garment. Solvent should not be used if it discolors the fabric. ${ }^{6}$

ii. An absorbent type white fabric, e.g. towel, is to be used under the spoiled fabric and stained item to be put back side front. Care should be taken for regular change of backed fabric to avoid migration of colour.

iii. In case of liquid stain allow for exuding before dry wash.

iv. Coloured towel as backing should be avoided to minimize the chance of migration of dye stain. Similarly stay away from linty clothes or paper.

v. After indentifying the affected spot dry cleaning to be followed according to the instruction written on fabric care label. Generally non-washable items are to be considered for solvent cleaning, viz. leather, fur suede etc. ${ }^{7}$

vi. Heat polymerizes the stain, so hot press should be avoided.

vii. Washing to be done after complete evaporation or rinsing out of dry cleaning agent. Properly ventilated laundering room is one of the criteria for such cleaning.

viii. Following the care label instruction stains are to be removed stepwise. Avoid drier until it is not confirmed that blotch is removed or not, if possible always action to be taken when stains are fresh. ${ }^{8}$

ix. In case of blended fabric select stain removal chemicals judiciously following the fibre sensitiveness over that remover.

x. Always water to be used should be from known source especially for hot water.

xi. Complete laundering to be done after removal of stain, both the fabric and residual solvent.

xii. Chlorine bleach is not to follow in case of silk, spandex, polyurethane and wool fabric.

xiii. 'Line dry process' should be followed during drying after complete stain removal.

xiv. Rigorous frictional washing may cause damage of fabric finish or fibre pilling. Also the process may spread stain or cause discoloration.

\section{Advantages of Triexta fabric made garment}

a) Washer-man efforts in cleaning are less. Just dip and wash without rigorous rubbing.

b) Drape quality of those parts where Triexta is used are much better than $100 \%$ cotton. So hanging property of garment is enhanced. c) Unlike using of soil repellent fabric there is no manufacturing related problem is encountered for Triexta fabrics.

d) The garment gives wearer comfort as the fabric is very soft in nature so abrasion related problem has not been noted from the users.

e) Due to Trieste's bio-friendly nature, garment is very much sustainable in the contemporary scenario.

The main downside of Triexta fibre is that, it is not in position to endorse the producer's averment on its features due to lack of history. Triexta is in the market since 8-9 years where as average lifecycle of a carpet is around 10-15 years. So it is too early to comment about the performance of the fibre, where as nylon is well established in the carpet market. Though Triexta is new comer but put its foot print in the Fashion garment industry in an accepted way, showing a declaration that it will roam in the next 10 years as claimed by DuPont and Mohawk. Due to its softness and durability it can be predicted that it will be sustainable as 'Future of Fibre' over others.

\section{Future steps}

Though Triexta has no past history of sustainability like polyester and nylon since $1-15$ years but its properties proved that it comes to stand and meet the demand of users in fashion, apparel and carpet industries. But it is not so still popular in garment textile fields. Manufacturers are not so comfortable to use this beautiful fibre in fabric form in their products. ${ }^{9}$

Our effort was that to show the benefits of the fabric in using day to day life. It has been proved that the fabric is comfortable to wear as well as wash friendly. This paper has proved that common domestic stains like, hot coffee, mustard and iodine can easily be washed by simple hot water and can be dried within a short span of time. Also stain resistance property of PTT makes itself a zero maintenance and almost needless to substitute product.

The studies like dye fading, piling, breathability etc. to be executed later which will establish the concept an undaunted acceptance from the fashion lovers.

\section{Acknowledgments}

None.

\section{Funding}

None.

\section{Conflicts of interest}

The authors declare that they have no competing interests.

\section{References}

1. Triexta fiber (Polytrimethylene Terephthalate).

2. Vasant Kothari. New-fibres-for-home-textile-PTT-Triexta-fibre.

3. Cheryl Simmons. Triexta PTT carpet fiber. 2019.

4. Pet my carpet. 
5. Wang J, Pei L, Ge H, et al. Textile finish's impact on fabric stain removal and whiteness maintenance during home laundry. 2013.

6. Beavers EJ, Stone J. Quick 'n easy stain removal. Iowa State University cooperative extension service; 1986.

7. Johnson Wax. Laundry products form ED2-11. Shout; 1989.
8. Maytag. Stain Removal Guide form number 19 YG1087.

9. North Central Regional Cooperative Extension. Stain removal for washable fabrics. Extension Services of Illinois, Michigan, Minnesota, Missouri, Nebraska, North Dakota, South Dakota, and Wisconsin; 1979. 